PROCEEDINGS OF THE

AMERICAN MATHEMATICAL SOCIETY

Volume 139, Number 11, November 2011, Pages 4113-4119

S 0002-9939(2011)10815-3

Article electronically published on March 25, 2011

\title{
TWO REMARKS ON THE LENGTH SPECTRUM OF A RIEMANNIAN MANIFOLD
}

\author{
BENJAMIN SCHMIDT AND CRAIG J. SUTTON
}

(Communicated by Jianguo Cao)

\begin{abstract}
We demonstrate that every closed manifold of dimension at least two admits smooth metrics with respect to which the length spectrum is not a discrete subset of the real line. In contrast, we show that the length spectrum of any real analytic metric on a closed manifold is a discrete subset of the real line. In particular, the length spectrum of any closed locally homogeneous space forms a discrete set.
\end{abstract}

\section{INTRODUCTION}

It is widely believed that a closed Riemannian manifold of dimension at least two has infinitely many prime closed geodesics. The results of numerous authors verify this belief for large classes of manifolds as summarized in [Ta, Section 5].

While considering this problem, Abraham introduced the notion of a bumpy metric $[\mathrm{A}$. The main result of $[\mathrm{A}$ ] is that the collection of smooth bumpy metrics on a closed manifold forms a residual set in the space of all smooth metrics on $M$ (see An for a complete proof); he conjectured that such metrics should have infinitely many prime closed geodesics. Abraham also showed that if $g$ is a bumpy metric on a closed manifold $M$, then for any $L>0$ the collection of its geometrically distinct closed geodesics of length less than $L$ is finite. Hence, we see that, generically, the length spectrum - the collection of lengths of all smoothly closed geodesics - is a discrete subspace of the real line.

To our knowledge, examples of closed manifolds with non-discrete length spectrum do not appear in the literature, motivating the following:

1.1. Proposition. Every closed manifold $M$ of dimension at least two admits a smooth metric with respect to which the length spectrum is a non-discrete subset of the real line. Specifically, let $\gamma: S^{1} \rightarrow M$ be a smooth simple closed curve in $M$, then the following holds.

(1) If the dimension of $M$ is at least three, then there is a smooth metric $g$ on $M$ with respect to which $\gamma$ is a closed geodesic and its length $l_{g}(\gamma)$ is an accumulation point of the length spectrum.

Received by the editors June 23, 2010 and, in revised form, September 27, 2010.

2010 Mathematics Subject Classification. Primary 53C22.

The first author's research was partially supported by NSF grant DMS-0905906.

The second author's research was partially supported by NSF grant DMS-0605247 and a Career Enhancement Fellowship from the Woodrow Wilson National Fellowship Foundation.

(C)2011 American Mathematical Society

Reverts to public domain 28 years from publication 
(2) If the dimension of $M$ is two and $\gamma$ is such that its tubular neighborhood is an orientable disk bundle (such curves exist), then the same result holds.

In addition, we note in Example 2.3 that the length spectrum can even be an uncountable subset of the real line.

Given Abraham's bumpy metric theorem and the previous proposition, one is led to consider whether there are additional large classes of metrics for which the length spectrum is discrete. For a real analytic manifold $M$, the dense subset of real analytic metrics in the space of smooth metrics forms such a class:

1.2. Proposition. Let $M$ be a closed real analytic manifold and $g$ a real analytic metric on $M$. Then the length spectrum of $(M, g)$ forms a discrete subset of the real line.

In closing, we note that it is a long-standing problem to determine whether the length spectrum of a Riemannian manifold $(M, g)$ is determined by the spectrum of its Laplace-Beltrami operator $\Delta$. In support of an affirmative answer to this question we note that the spectrally determined tempered distribution Trace $\left(e^{i t \sqrt{\Delta}}\right)$ has its singular support contained in the length spectrum of $(M, g)$ (see $\mathrm{DuGu}$, Corollary 1.2] and [Ch, Theorem I]). In fact, for each $p=0,1, \ldots, \operatorname{dim}(M)$, the singular support of the tempered distribution Trace $\left(e^{i t \sqrt{\Delta_{p}}}\right)$ is a subset of the length spectrum, where $\Delta_{p}$ is the Laplacian on $p$-forms (see [DuGu, p. 40]). Thus, for each $p=0,1, \ldots, \operatorname{dim}(M)$, the spectrum of $\Delta_{p}$ determines a particular subset of the length spectrum and generically we have equality. In stark contrast, we note that Miatello and Rossetti have constructed examples of flat manifolds that are isospectral on 1-forms but that do not share the same length spectrum MR. Hence, in general, the singular support of Trace $\left(e^{i t \sqrt{\Delta_{1}}}\right)$ is a proper subset of the length spectrum.

Now we recall that the covering spectrum of a Riemannian manifold, denoted by $\operatorname{CovSpec}(M, g)$, is a geometric invariant that, roughly speaking, measures the circumference of certain holes in a Riemannian manifold $(M, g)$ by isolating the lengths of certain closed geodesics [SW]. In particular, $\operatorname{CovSpec}(M, g)$ is a subset of the non-negative reals such that $2 \cdot \operatorname{CovSpec}(M, g)$ is contained in the length spectrum of $(M, g)$ and any $\ell$ appearing in the set $2 \cdot \operatorname{CovSpec}(M, g)$ is the length of a closed geodesic that is shortest in its free homotopy class. Sormani and Wei noted that if $\left\{g_{t}\right\}_{t \in \mathbb{R}}$ is a one-parameter family of Riemannian metrics on a smooth manifold $M$ having a common discrete length spectrum, then they have a common covering spectrum [SW, Corollary 8.8]. That is, among manifolds with discrete length spectrum, the covering spectrum is an invariant of "iso-length-spectral" deformations. Hence, in light of the previous discussion and the fact that the covering spectrum is not a spectral invariant [DGS], it would seem to be an interesting question to examine whether the covering spectrum is fixed for a one-parameter family of isospectral analytic manifolds.

\section{THE PROOFS}

Proof of Proposition 1.1. Let $M$ be a closed manifold of dimension $n \geq 3$, let $S^{1}=$ $\mathbb{R} / \mathbb{Z}$ denote the standard circle and let $\gamma: S^{1} \rightarrow M$ be a smooth simple closed curve contained in the interior of $M$. The tubular neighborhood theorem $\mathrm{Hi}$, Thm. 5.2, Ch. 4] says that there is a smooth vector bundle $N$ over $S^{1}$ of rank $n-1$ along 
with a diffeomorphism $i: N \rightarrow \mathcal{T} \subset M$ onto an open subset $\mathcal{T}$ of $M$ so that its composition with the zero section $S^{1} \rightarrow N$ is the map $\gamma$.

We now recall that up to isomorphism there are exactly two vector bundles of rank $n-1$ over $S^{1}$ : one orientable and one non-orientable [Hi, Ch. 4, Sec. 4, Ex. 2], [Ra, Chp. 5]. To facilitate constructing the metric $g$, we let $B^{n-1}$ be the standard open unit ball around 0 in $\mathbb{R}^{n-1}$, which is diffeomorphic to each fiber of the vector bundle $N$ over $S^{1}$. Now consider the quotient $\left(B^{n-1} \times \mathbb{R}\right) / \mathbb{Z}$, where $z \in \mathbb{Z}$ acts by sending $\left(\left(x_{1}, x_{2}, \ldots, x_{n-1}\right), t\right)$ to $\left(\left(x_{1}, x_{2}, \ldots, x_{n-1}\right), t+z\right)$ if $N$ is orientable, and to $\left(\left((-1)^{z} x_{1}, x_{2}, \ldots, x_{n-1}\right), t+z\right)$ if $N$ is non-orientable. We then have the following commutative diagram:

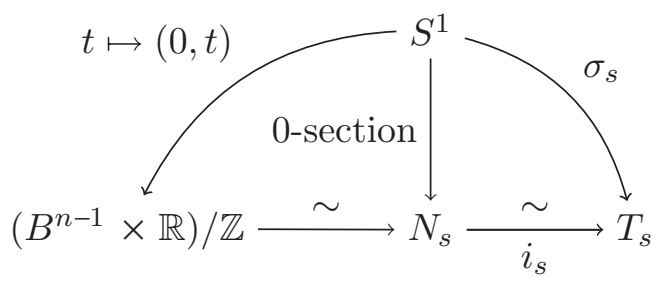

where the horizontal maps are diffeomorphisms.

The standard metric $h_{0}=h_{0}^{B} \oplus h_{0}^{\mathbb{R}}$ on $B^{n-1} \times \mathbb{R}$ induces a Riemannian metric on $\mathcal{T}$ that we also denote by $h_{0}$. Now, let $\left\{a_{k}\right\}_{k \geq 2}$ be a sequence of numbers greater than 1 so that $a_{k} \rightarrow 1$, and for each $k \geq 2$, let $c_{k}=\left(0, \frac{1}{k}, 0, \ldots, 0\right) \in B^{n-1}$, $\epsilon_{k}=\frac{1}{2 k(k+1)}$ and let $B_{k}=B\left(c_{k} ; \epsilon_{k}\right)$ be the ball of radius $\epsilon_{k}$ centered at $c_{k}$. Then for each $k \geq 2$ we let $f_{k}: B_{k} \rightarrow\left[1, a_{k}\right]$ be a smooth (radially symmetric) bump function with $f_{k} \equiv a_{k}$ on $B\left(c_{k} ; \frac{1}{4} \epsilon_{k}\right)$ and $f_{k} \equiv 1$ on $B\left(c_{k} ; \epsilon_{k}\right)-B\left(c_{k} ; \frac{3}{4} \epsilon_{k}\right)$. Now, choosing the sequence $\left\{a_{k}\right\}$ so that $a_{k}$ converges to 1 sufficiently fast, we obtain a smooth bounded function $f: B^{n-1} \rightarrow \mathbb{R}$ given by

$$
f(x)= \begin{cases}1 & x \in B^{n-1}-\bigcup_{k \in \mathbb{N}} B_{k} \\ f_{k}(x) & x \in B_{k} .\end{cases}
$$

We may then define a $\mathbb{Z}$-invariant metric $h$ on $B^{n-1} \times \mathbb{R}$ via

$$
h(x ; t)=\left(h_{0}^{B}\right)_{x} \oplus f(x)\left(h_{0}^{\mathbb{R}}\right)_{t} .
$$

Then $h$ induces a smooth metric, which we also denote by $h$, on the tubular neighborhood $\mathcal{T}$.

For each $k \geq 2$, let $\gamma_{k}: S^{1} \rightarrow \mathcal{T}$ be the smoothly closed loop induced by the smooth curve $\tilde{\gamma}_{k}: \mathbb{R} \rightarrow B^{n-1} \times \mathbb{R}$ given by $t \mapsto\left(c_{k} ; t\right)$. Then with respect to the metric $h$ we see that $\gamma_{k}: S^{1} \rightarrow(\mathcal{T}, h)$ is a geodesic with $l_{h}\left(\gamma_{k}\right)=a_{k}$. Now, let $r: B^{n-1} \rightarrow[0, \infty)$ be the smooth map given by $r(x)=\|x\|^{2}$ and for any $\epsilon>0$, let $\mathcal{T}_{\epsilon} \equiv\{\overline{(x ; t)} \in \mathcal{T}: r(x)<\epsilon\}$. We then obtain a smooth metric $g$ on $M$ given by

$$
g_{p}= \begin{cases}\left(\theta(r(x)) h+(1-\theta(r(x))) g_{0},\right. & p=\overline{(x ; t)} \in \mathcal{T}, \\ g_{0}, & p \in M-\mathcal{T},\end{cases}
$$

where $g_{0}$ is some background metric on $M$ and $\theta:[0,1] \rightarrow[0,1]$ is smooth with $\theta(t)=1$ for $0 \leq t \leq 2 / 5$ and $\theta(t)=0$ for $3 / 5 \leq t \leq 1$. Then on $\mathcal{T}_{2 / 5}$ we see that $g=h$. Hence, $\gamma: S^{1} \rightarrow(M, g)$ is a geodesic with $l_{g}(\gamma)=l_{h}(\gamma)=1$ and, for all $k \geq 2, \gamma_{k}: S^{1} \rightarrow(M, g)$ is a closed geodesic (freely homotopic to $\gamma$ ) with $l_{g}\left(\gamma_{k}\right)=\bar{l}_{h}\left(\gamma_{k}\right)=a_{k}$. Hence, $l_{g}(\gamma)$ is an accumulation point of the length spectrum of $(M, g)$. 
In dimension two, if one assumes that the tubular neighborhood $\mathcal{T}$ of $\gamma$ is orientable (this holds for any $\gamma$ contained in a coordinate chart), then the above construction carries through without modification.

2.1. Remark. The argument remains true for an arbitrary smooth manifold $M$ of dimension at least two. In the case where $M$ has boundary, one just needs to choose $\gamma: S^{1} \rightarrow M$ for which the image is in the interior of $M$. However, in the case of a compact manifold with boundary, one is usually more interested in the closed orbits of the billiard ball map.

2.2. Remark. Since the length $l_{g}(\gamma)$ in Proposition 1.1 is an accumulation point of the length spectrum of $g$, it follows from [DuGu, Theorem 4.5] that $l_{g}(\gamma)$ is an "unclean" length. That is, $l_{g}(\gamma)$ does not satisfy the so-called "clean interaction hypothesis" of [DuGu, Theorem 4.5]. Consequently, the analysis of Duistermaat and Guillemin does not resolve whether the singular support of the wave trace is equal to the length spectrum for the metrics given by Proposition 1.1. The following example can be used to construct a closed surface with uncountably many "unclean" lengths in its length spectrum.

2.3. Example (Uncountable Length Spectrum). Not only may the length spectrum fail to be discrete, it can even be an uncountable subset of $\mathbb{R}$. Indeed, let $K \subset[0,1]$ denote the standard middle third Cantor set and let $f:[0,1] \rightarrow \mathbb{R}$ be a nonnegative smooth function with zero set $K$. Then the function $g:[0,1] \rightarrow \mathbb{R}$ defined by $g(x)-1=\int_{0}^{x} f(t) d t$ has critical set $K$ and is strictly increasing on $K$. Consider the metric of revolution on $[0,1] \times S^{1}$ obtained by revolving the graph of $g$ around the $x$-axis. For each $k \in K$ the set $\{k\} \times S^{1}$ is a closed geodesic of length $2 \pi g(k)$. As $K$ is uncountable and $g$ increases on $K$, this metric has uncountable length spectrum.

Proof of Proposition 1.2. We begin by fixing some notation.

Let $\Omega$ be the set consisting of piecewise smooth loops in $M$. That is, $\omega:[0,1] \rightarrow$ $M$ is an element of $\Omega$ if and only if

(1) $\omega(0)=\omega(1)$;

(2) there is a subdivision $t_{0}=0<t_{1}<\cdots<t_{k}=1$ such that $\omega \uparrow\left[t_{i-1}, t_{i}\right]$ is smooth for each $i=0, \ldots, k-1$.

Given $\omega \in \Omega$, the tangent space to $\Omega$ at $\omega$, denoted by $T_{\omega} \Omega$, is the collection of all vector fields $W_{t}$ along $\omega$ which are smooth on the corresponding intervals $\left[t_{i-1}, t_{i}\right]$ for $i=1, \ldots, k$ and satisfy $W_{0}=W_{1}$. By a variation of $\omega$ (through closed loops) we shall mean a map $\bar{\alpha}:(-\epsilon, \epsilon) \rightarrow \Omega$ such that

(1) $\bar{\alpha}(0)=\omega$

(2) there is a partition $t_{0}=0<t_{1}<\cdots<t_{l}=1$ of $[0,1]$ such that the $\operatorname{map} \alpha:(-\epsilon, \epsilon) \times[0,1] \rightarrow \Omega$, given by $\alpha(u, t)=\bar{\alpha}(u)(t)$, restricted to $(-\epsilon, \epsilon) \times\left[t_{i-1}, t_{i}\right]$, is smooth for each $i=1, \ldots, l$.

Given any variation $\bar{\alpha}$ of $\omega$, the vector field $W_{t}=\frac{\partial}{\partial u} \alpha(0, t)$ is in the tangent space $T_{\omega} \Omega$, and conversely any vector field $W_{t} \in T_{\omega} \Omega$ gives rise to a variation of the loop $\omega$ as follows: $\alpha(u, t)=\exp _{\omega(t)}\left(u W_{t}\right)$.

We let $E: \Omega \rightarrow \mathbb{R}$ be the energy functional

$$
E(\omega) \equiv \int_{0}^{1}\left\|\omega^{\prime}(t)\right\|^{2} d t
$$


and let $L: \Omega \rightarrow \mathbb{R}$ be the length functional

$$
L(\omega)=\int_{0}^{1}\left\|\omega^{\prime}(t)\right\| d t
$$

The Cauchy-Schwarz inequality implies that $L(\omega)^{2} \leq E(\omega)$ with equality if and only if $\left\|\omega^{\prime}(t)\right\|$ is constant. In particular, equality is obtained when $\omega$ is a geodesic. The first variation formula implies that $\omega \in \Omega$ is a closed geodesic in $(M, g)$ if and only if it is a critical point of $E$; that is, $\frac{d}{d u}(E \circ \bar{\alpha})(0)=0$ for any variation $\bar{\alpha}$.

Now for each $c>0$ we let $\Omega^{c}=E^{-1}([0, c])$ and Int $\Omega^{c}=E^{-1}([0, c))$. The space $\Omega^{c}$ is infinite dimensional; however, following Milnor $[\mathrm{M}]$ we can construct a finite dimensional approximation to $\Omega^{c}$ as follows.

For each partition $t_{0}=0<t_{1}<\cdots<t_{k}=1$ of the unit interval $[0,1]$ we let $\Omega\left(t_{0}, t_{1}, \ldots, t_{k}\right)$ be the collection of $\omega:[0,1] \rightarrow M$ such that

(1) $\omega(0)=\omega(1)$

(2) $\omega\left\lceil\left[t_{i-1}, t_{i}\right]\right.$ is a geodesic for $i=0,1, \ldots, k-1$.

That is, $\Omega\left(t_{0}, t_{1}, \ldots, t_{k}\right)$ is the space of piecewise geodesic loops. We let

$$
\Omega\left(t_{0}, t_{1}, \ldots, t_{k}\right)^{c}=\Omega^{c} \cap \Omega\left(t_{0}, t_{1}, \ldots, t_{k}\right)
$$

and

$$
\operatorname{Int} \Omega\left(t_{0}, t_{1}, \ldots, t_{k}\right)^{c}=\left(\operatorname{Int} \Omega^{c}\right) \cap \Omega\left(t_{0}, t_{1}, \ldots, t_{k}\right) .
$$

Then, as in Milnor, we have the following lemma.

2.4. Lemma (cf. $\mathrm{M}$, Lemma 16.1 and Theorem 16.2). Let $(M, g)$ be a compact real analytic Riemannian manifold and let $c>0$. Then for all finite partitions $t_{0}=$ $0<t_{1}<\cdots<t_{k}=1$ with sufficiently small mesh, the set $B \equiv \operatorname{Int} \Omega\left(t_{0}, t_{1}, \ldots, t_{k}\right)^{c}$ can be given the real analytic structure of an open subset of $M^{k}$ in a natural way. Furthermore, the restriction of the energy functional $E$ to $B$, which we will denote by $\widetilde{E}$, has the following properties:

(1) $\widetilde{E}$ is analytic and given by $\widetilde{E}(\omega)=\sum_{i=1}^{k} \frac{d_{g}\left(\omega\left(t_{i-1}\right), \omega\left(t_{i}\right)\right)^{2}}{\left(t_{i}-t_{i-1}\right)}$;

(2) for each $0<a<c$, the set $B^{a} \equiv \widetilde{E}^{-1}([0, a])$ is a compact subset of $B$;

(3) the critical points of $\widetilde{E}$ on $B$ coincide with those of $E$ on $\operatorname{Int} \Omega^{c}$.

Proof. Since $M$ is compact we may fix an $\epsilon>0$ so that whenever $x, y \in M$ are such that $d_{g}(x, y)<\epsilon$, there is a unique minimizing geodesic from $x$ to $y$ of length less than $\epsilon$; and, since $g$ is analytic, this geodesic will depend analytically on $x$ and $y$ (cf. [M], Lemma 10.3]).

Now take a subdivision $t_{0}=0<t_{1}<\cdots<t_{k}=1$ of the interval $[0,1]$ with mesh less than $\epsilon^{2} / c$. Then for any $\omega \in \operatorname{Int} \Omega\left(t_{0}, t_{1}, \ldots, t_{k}\right)^{c}$ we have

$$
\begin{aligned}
d_{g}\left(\omega\left(t_{i-1}\right), \omega\left(t_{i}\right)\right)^{2}=\left(L_{t_{i-1}}^{t_{i}} \omega\right)^{2} & =\left(t_{i}-t_{i-1}\right) E_{t_{i-1}}^{t_{i}} \omega \\
& \leq\left(t_{i}-t_{i-1}\right) E \omega \\
& <\left(t_{i}-t_{i-1}\right) c \\
& <\epsilon^{2} .
\end{aligned}
$$

Therefore by our choice of $\epsilon$, the geodesic $\omega \uparrow\left[t_{i-1}, t_{i}\right]$ is uniquely and analytically determined by its endpoints, and we conclude that the map

$$
\omega \in B \stackrel{\phi}{\mapsto}\left(\omega\left(t_{0}\right), \omega\left(t_{1}\right), \ldots, \omega\left(t_{k-1}\right)\right) \in M^{k}
$$


defines a homeomorphism of $B$ onto an open subset of $M^{k}$, which induces a real analytic structure on $B$.

The expression for $\widetilde{E}$ given in (1) follows from Equation 2.5, and the analyticity follows since the geodesics depend analytically on the endpoints. Property (2) follows from the fact that $\phi$ maps $B^{a}$ homeomorphically onto the closed subset of $M^{k}$ given by

$$
\left\{\left(p_{0}, p_{1}, \ldots, p_{k-1}\right) \in M^{k}: \sum_{i=1}^{k-1} \frac{d_{g}\left(p_{i-1}, p_{i}\right)^{2}}{\left(t_{i}-t_{i-1}\right)}+\frac{d_{g}\left(p_{k-1}, p_{0}\right)^{2}}{\left(t_{k}-t_{k-1}\right)} \leq a\right\}
$$

which is compact since $M$ is compact. Finally, statement (3) follows from the fact that each smoothly closed geodesic is a broken geodesic and the first variation formula, which implies that the critical points of $\widetilde{E}$ are the smooth geodesics.

Now, assuming that the assumptions of the preceding lemma are obtained, for each $0<a<c, B^{a}$ is a compact subset of $B$ that contains all the critical points of $E$ on Int $\Omega^{a}=E^{-1}([0, a))$. We now recall the following theorem concerning the critical values of real analytic functions.

2.6. Theorem ([SS]). Let $f: D \subset \mathbb{R}^{N} \rightarrow \mathbb{R}$ be a real analytic function on an open subset of Euclidean $N$-space, and let $Z$ be the collection of critical points of $f$ on $D$. Then for any compact subset $K \subset D$ we have that $f(Z \cap K)$ is finite. That is, the set of critical values of $f$ restricted to a compact set is always finite.

It follows that since $\widetilde{E}: B \rightarrow \mathbb{R}$ has the same critical values as $E$ and $B^{a} \subset B$ is compact, the collection of critical values of $E$ on $\operatorname{Int} \Omega^{a}=E^{-1}([0, a))$ is finite. That is, there are finitely many critical values of $E$ less than $a$. Therefore, the length spectrum of $(M, g)$ is a discrete subset of $\mathbb{R}$.

2.7. Remark. As a consequence of Proposition 1.2, a locally homogeneous Riemannian manifold has a discrete length spectrum, our original motivation for this paper. A nearly identical proof gives the following analogue of Proposition 1.2 for noncompact real analytic manifolds: If $M$ is a real analytic manifold, then an infinite set of closed geodesics with accumulating lengths cannot accumulate in $M$.

\section{REFERENCES}

[A] R. Abraham, Bumpy metrics, Global Analysis (Proc. Sympos. Pure Math., Vol. XIV, Berkeley, Calif., 1968), 1-3, Amer. Math. Soc., Providence, R.I., 1970. MR0271994 $(42: 6875)$

[An] D.V. Anosov, Generic properties of closed geodesics, Math. USSR Izvestiya 21 (1983), 1-29. MR670163 (84b:58029)

[Ch] J. Chazarain, Formule de Poisson pour les variétés riemanniennes, Invent. Math. 24 (1974), 65-82, MR0343320 (49:8062)

[DGS] B. De Smit, R. Gornet and C. J. Sutton, Sunada's method and the covering spectrum, J. Differential Geom. 86 (2010), no. 3, 501-537.

[DuGu] J.J. Duistermaat and V. Guillemin, The spectrum of positive elliptic operators and periodic bicharacteristics, Invent. Math. 29 (1975), 39-79. MR0405514 (53:9307)

[Hi] M.W. Hirsch, Differential topology, Graduate Texts in Math., 33, Springer-Verlag, New York (1976). MR0448362 (56:6669)

[MR] R. J. Miatello and J. P. Rossetti, Length spectra and p-spectra of compact flat manifolds, J. Geom. Anal. 13 (2003), 631-657. MR2005157 (2005a:58053)

[M] J. Milnor, Morse Theory, Annals of Mathematics Studies, 51, Princeton University Press, Princeton (1969). MR0163331(29:634) 
[Ra] A. Ranicki, Algebraic and geometric surgery, Oxford University Press, New York (2002). MR2061749 (2005e:57075)

[SW] C. Sormani and G. Wei, The covering spectrum of a compact length space, J. Differential Geom. 67 (2004), 33-77. MR2153481 (2006e:58050)

[SS] J. Souček and V. Souček, Morse-Sard theorem for real-analytic functions, Comment. Math. Univ. Carolinae 13 (1972), 45-51. MR0308345 (46:7459)

[Ta] I.A. Taimanov, The type number of closed geodesics, Regular and Chaotic Dynamics 15 (2010), 84-100. MR2593232

Department of Mathematics, Michigan State University, East Lansing, Michigan 48824

E-mail address: schmidt@math.msu.edu

Department of Mathematics, Dartmouth College, Hanover, New Hampshire 03755

E-mail address: craig.j.sutton@dartmouth.edu 